\title{
Mental health of the ageing immigrant population
}

\author{
Gill Livingston \& Sati Sembhi
}

Abstract About $6 \%$ of older people in the UK are immigrants. Concentrated in deprived inner-city areas, their numbers are rising rapidly, with the ageing of those arriving after the Second World War. Cultural, language and educational differences cause problems in studying this group's mental health. Idioms of distress may affect presentation, help-seeking behaviour and acceptability of treatment. Ethnic elders may be considered vulnerable to depression because of socio-economic deprivation, immigrant status and old age but studies are contradictory and may use inappropriate screening instruments. Relatively few consider immigrant status and dementia. Uncontrolled hypertension could relate to higher dementia rates in Black immigrants which are not reflected in the country of origin. No genetic risk has been found. There is potential for prevention in this population.

This article concerns the mental health of older people who have moved from their country of birth - the older immigrant population. In the UK, although the absolute numbers of ethnic elders are relatively small (about $6 \%$ of people aged 65 and over were born outside the country), there is a greater concentration of pensioners from ethnic minorities in deprived inner-city areas. Numbers are also increasing rapidly, as those who immigrated to the UK after the Second World War reach retirement age.

It is important to be aware of immigrant groups, not only for adequate and culturally appropriate provision of services, but also because immigrant status can hold important clues to the aetiology of illnesses. It has been hypothesised that relative socio-economic deprivation, ageing and immigrant status - the 'triple whammy' - lead to a particular vulnerability to mental illness in these groups (Rait et al, 1996). Although there have been more studies of older immigrants in recent years, there is a relative lack of research.

\section{Pitfalls of studying immigrant people}

There are considerable difficulties in studying immigrant and ethnic populations. In addition to personal factors intrinsic to being a new immigrant
(Box 1), we should consider the effect of the new country of residence on immigrants. There is little explicit consideration of the changes that occur when immigrants live in a new culture. It is often assumed that they remain unaffected by the experience. In fact, immigrants interact and adapt to their new environment, undergoing a degree of acculturation and sometimes assimilation. Furthermore, the meaning of the move, the welcome (or lack of it) on arrival and the age and stage of life at which people move make for different experiences, even when people originate from the same culture. Much of the research into the effect of immigration focuses on increased vulnerability rather than on the resilience and protection that may be afforded by, for example, close social groups.

\section{Box 1 Difficulties in studying immigrant populations}

Misinterpretation of responses because of cultural difference, language and education

Idioms of distress may affect presentation, helpseeking behaviour, likelihood of diagnosis and acceptability of treatment

Unjustifiable assumption of homogeneity of people from a single large geographical area

Varying reasons for immigration, e.g. education, asylum, employment

Gill Livingston is a reader in the psychiatry of old age at Royal Free and University College Medical School (Department of Psychiatry and Behavioural Sciences, Wolfson Building, 48 Riding House Street, London W1N 8AA, UK. Tel: 02075302309 ; fax: 0207530 2304; e-mail: g.livingston@ucl.ac.uk) and an honorary consultant in mental health care of older people for Camden and Islington Mental Health Trust. Her research interests include the epidemiology of psychiatric illness in older people and the mental health of carers and ethnic elders. Sati Sembhi is a consultant psychiatrist in Auckland, New Zealand. She completed dual training in old age and general adult psychiatry in London. Her research interests are explanatory models of illness, use of non-orthodox therapies and mental health of ethnic elders. Her ongoing research investigates experiences of older immigrants moving to the UK. 


\section{Defining race and ethnicity}

The definition of race and ethnicity (Box 2) as research variables is contentious. An 'ethnic group' may share geographical origins, culture, religious traditions and language. 'Culture' is the network of shared ideas, meaning and rules, a lens through which a person perceives and understands the world (Helman, 2000: p. 2). 'Ethnicity' therefore refers to a self-described identity. As people see themselves in different ways at different times, it is not a fixed concept. When immigrants' children are subjects of research, the study of ethnicity becomes more difficult, as classification becomes even less clear. People from ethnic minorities are also increasingly likely to marry people from the indigenous population or those from a different ethnic minority. Ethnicity can also refer to imposed categories such as those in the 1991 UK census, which has been used for much of the UK research but did not allow a mixed ethnicity category. Many people found it difficult to classify themselves. 'Race' is a term implying genetic homogeneity, and racial distinctions are generally based on physical appearance largely skin colour. It gives little information about immigrant status or ethnic group.

As immigrant groups establish themselves, they may wish to continue a lifestyle based on cultural or religious traditions from their country of origin and hope to pass this on to the next generation. Tensions may arise if children do not want to adopt the way of life preferred by their parents. This can become particularly apparent at important times in the life cycle, such as marriage and childbirth, which are traditionally times when families come together. There may also be problems as older immigrants become more frail and dependent.

\section{Assessing dementia (Box 3)}

Cross-cultural assessment of dementia in older people has specific pitfalls related to language and literacy skills. In particular, the use of culturally biased screening instruments that rely on language recognition and familiarity with test situations may be inappropriate or misleading for people with cognitive impairment (Lindesay, 1998). Culturally appropriate norms are also important in the evaluation of dementia. Immigrants may be literate in a different language or functionally illiterate. Recent immigrants from rural areas may have had little need for the concept of complex maps and exact dates. As a result, interpreting cognitive testing without a knowledge of education and background is likely to lead to errors in diagnosis. It may be important, therefore, to validate and modify instruments for examining cognitive function in different ethnic

\section{Box 2 Defining race and ethnicity}

Race

- The term often implies genetic homogeneity

- Racial distinctions are often made on the basis of appearance, particularly skin colour

Ethnicity

- This is self-described, onthe basis of shared origins, culture, religion and language

- Self-description of ethnicity is not fixed

- Forced choice of ethnic category (e.g. as in the 1991 UK census) may lead to oversimplification and falsification

groups. One report of validation of the Mini-Mental State Examination (MMSE) in South Asians in the UK found that cut-offs varied when judged against a diagnostic instrument for the detection of dementia (Rait et al, 2000). A new normative test battery for cognitive function in African-Caribbean elders in the UK shows that, compared with normative data for the African American population, scores on verbal fluency were lower but scores on memory tests were comparable (Stewart et al, 2001b).

Despite these caveats, a 1-year follow-up of a multicultural community sample of people with a diagnosis of dementia found that stability of diagnosis did not vary according to ethnic background (Schofield et al, 1995). Similarly, a small study of Gujarati people diagnosed with dementia found that the diagnosis was stable at follow-up (Shah et al, 1998).

\section{Assessing depression}

Depression may present with different patterns of symptoms in different cultures. People of some cultures commonly present with predominant

\section{Box 3 Key learning points: dementia}

Relatively few studies consider immigrant status as a risk factor for dementia

Recent studies suggest that dementia may be increased in Black immigrants

Increased rates of dementia in Black immigrants do not appear to reflect increased rates in the country of origin

Excess of hypertension in African-Caribbean populations in the West is well documented

Excess of dementia may be related to uncontrolled hypertension and diabetes

There is potential for primary and secondary prevention in this population

APOE 4 allele frequency does not seem to vary between populations but expression may be decreased in African and Hispanic Americans 
symptoms of sadness and low mood during depression, whereas others present with pain and somatic symptoms. The language of distress used, for example, by African Americans differs from that on which structured diagnoses are made and may lead to an underestimate of distress and anxiety levels. When screening for depression in older people, lower cut-points have been found to be appropriate for older Black people living in the UK (Abas et al, 1998).

\section{Current research evidence}

Much of our current knowledge of mental illness in immigrant elders relies on research carried out in the USA, where the experience of immigration may differ from that in the UK. The USA sees itself as a melting pot and it is proud of the idea that economic migrants come to strive for, and find, success. In contrast, the term 'economic migrant' is used in Britain as a term of abuse, with implications in parts of the media that people are coming here to live off the state. Conversely, African Americans are descendants of an extremely oppressed population, subject to overt racism. The studies from the USA are therefore of a population that is different from that in the UK, and comparable studies between the two countries may have different results.

\section{Dementia in older immigrants}

\section{Prevalence of dementia}

The overall prevalence of dementia within the population is usually found to be between $5 \%$ and $6 \%$ of those aged 65 years and above, living at home. Metaanalysis (Jorm et al, 1987) has shown that the prevalence doubles with every 5-year increase in age band up to around age 90 years. Most studies have found that education is protective against both the development and the progression of dementia. Migrant groups with relative socio-economic deprivation, often associated with less education, might therefore be expected to be more vulnerable to dementia. The relative youth of the ageing immigrant population compared with the ageing indigenous population would then decrease the overall prevalence.

Few studies consider whether ethnicity or immigrant status are risk factors for dementia and most that do are small and lack concurrent controls. For example, one comparison of Black with White community residents found that, out of a total of 26 people with dementia, Black older people were significantly more likely to have dementia than White $(16 \%$ v. 3\%) and that there was an excess history of stroke, diabetes and hypertension in the Black population.
Another small study (14 people in the total population had dementia) surveyed English-speaking Black and White residents in the USA. The ageadjusted prevalence of dementia in Black men was found to be almost twice that of White men $(4.8 \% v$. $2.4 \%$; Perkins et al, 1997), although the difference was not statistically significant.

In the UK, 418 older ethnic elders living in Liverpool were interviewed (McCracken et al, 1997). There was a high prevalence of dementia in the ethnic minority population as a whole, particularly in Black African groups, when compared with an earlier study using the same instruments. The authors concluded that these results could be attributed to the effects of participants' age, gender and inability of some to speak English and that there was no true increase in community prevalence in the ethnic minority population. Another UK study examined South Asian older residents and found an increased rate of dementia compared with similar but not directly comparable populations using the same instrument although, again, no direct comparisons were made (Bhatnagar \& Frank, 1997). Language difficulties may have affected results in this study, as residents were interviewed in Hindi, although this was not necessarily their first language. This may account for the fact that the concordance of the diagnosis from the study with psychiatric diagnosis was very low.

A more recent UK pilot study, however, suggested a higher rate of dementia in African-Caribbeans than in age- and gender-matched White residents (Richards et al, 2000). Our own study found that the prevalence of dementia was raised in immigrant African-Caribbeans compared with White and ethnic minority UK-born individuals (17.3\%, RR = $1.7,95 \%$ CI 1.1-2.8), despite the fact that those of African-Caribbean origin were significantly younger (Livingston et al, 2001a). In this study, the findings did not appear to arise either through language or differing education.

The excess of hypertension in the AfricanCaribbean population in the West is well documented, as is an increased mortality in this group from cerebrovascular disease. Despite the known excess of hypertension in this population, we did not find an excess of self-reported hypertension or of those taking antihypertensive medication, although there was an excess of self-reported diabetes.

Hendrie et al (2001) first compared the prevalence of dementia in community-dwelling people living in Nigeria and in African Americans living in Indianapolis, USA, and found a significantly decreased rate in Nigeria $(2.3 \%$ v. $4.8 \%)$. A recent follow-up study compared the incidence rates of dementia in the two populations: the Yoruba in Nigeria and agestandardised African Americans. The Yoruba group showed a significantly lower incidence rate of 
Alzheimer's disease (1.2\% v. 2.5\%) and dementia $(1.4 \%$ v. 3.2\%) (Hendrie et al, 2001). This suggests that the development of dementia is influenced by changes associated with moving from Nigeria to live in the USA. The investigator felt that there was potential to identify modifiable environmental and genetic factors by further comparison of those two groups. A similar picture emerged in a comparison of Indian residents in India with Indian residents in Pennsylvania, USA, where prevalence rates for Alzheimer's disease among those aged 70-79 years was $0.7 \%$ v. 3.1\% (Ganguli et al, 2000).

\section{Dementia and the APOE 4 allele}

Most studies have found that the strongest genetic risk factor for the development of Alzheimer's disease is the possession of the apoliprotein E (APOE) 4 allele. The allele frequency does not vary between Black Americans, Hispanic Americans and White Americans. The possession of the allele has, however, been found to have a weaker association with Alzheimer's disease in some populations, including African Americans, Caribbean-Hispanics in the USA and native Spanish people, although not in Indians (Ganguli et al, 2000).

One meta-analysis of studies found that, overall, the association between possession of the APOE 4 allele and the development of Alzheimer's disease was lower for African and Hispanic Americans than for Whites but higher for Japanese people (Farrer et $a l, 1997)$. As the individual studies reported a heterogeneous effect of the allele, in particular with regard to African Americans, the true effect requires further clarification.

\section{Pattern, presentation and course of dementia}

Although, as we have already discussed, sociocultural factors such as education and language may influence the time of presentation and pattern of symptoms in dementia, there have been few reports of any differences. In different cultures, the meaning of memory difficulties and dependency may differ, so that if older people are expected to be less independent, the symptoms of early dementia may not be regarded as pathological. Those who are supported by their families and communities may present relatively early, as change is noticed in them. Those without social support may present late. The lack of insight, apathy and decreased ability to express and organise in those with dementia mean that they lack their own voice and require an advocate.

A study comparing Black and White Americans with Alzheimer's disease found that Black patients had fewer years of education, were more likely to have hypertension and reported shorter duration of illness at the time of presentation but had lower MMSE scores (Hargrave et al, 1998). They also reported more insomnia but less anxiety. A study of consecutive admissions of older African Americans compared with White people with dementia found, however, no difference in behavioural disturbance presented or response to treatment between the two groups (Akpaffiong et al, 1999). Ethnic differences have been found to have little effect on mortality rates (Jolley \& Baxter, 1997)

\section{Depression (Box 4)}

In most studies in the USA, Black and White elders show no difference in rates of depression (e.g. Blazer et al, 1996). In the UK, rates of depression and anxiety have been found to be the same (e.g. Lindesay et al, 1997) or slightly higher in Black African elders (McCracken et al, 1997). The latter study found that Black Africans with depression were less likely to see relatives, and the authors considered that this was significantly associated with depression. In east London, older people of Bengali and Somalian ethnicity were found to have a surprisingly high but varying prevalence of depression (77\% and $25 \%$, respectively) when compared with two different control populations of White people (5\% and $25 \%$ ) (Silveira \& Ebrahim, 1998a, 1998b). However, the populations were sampled in very different ways: the Somali population was from a lunch club, the Whites from an age-gender register. The same study found that Gujaratis had a lower prevalence of depression ( $2 \%)$. When adjusted for age, income, physical health and social problems, ethnicity was no longer a significant risk factor for high depression scores.

\section{Box 4 Key learning points: depression}

Ethnic elders are thought to be particularly vulnerable to depression because of risk factors of socio-economic deprivation, immigrant status and old age

Studies are contradictory

Most studies do not describe potential confounders such as physical ill health, living alone and social class

Screening instruments validated in a mainly White older population may not be valid for other populations

Vascular hypothesis suggests that depression in old age is caused by vascular pathology but recent studies suggest an association with stroke but not vascular risk factors 
Another study of older people of South Asian origin estimated a $20 \%$ prevalence of depression (Bhatnagar \& Frank, 1997). A study in north London showed that the mental health of Hindu grandmothers was associated with family structure, with those coming from nuclear rather than extended families being more susceptible to depression (Guglani et al, 2000).

For African-Caribbean elders, in particular, rates based on community screening using instruments with cut-offs validated on predominantly White populations have been found to be underestimates (Abas et al, 1998). The Irish population in general, although not older people in particular, has been shown to have poorer mental and physical health than other ethnic minority groups. They have the highest rates of hospital admissions for mental illness and high rates of suicide and attempted suicide (Merril \& Owens, 1988).

Our own recent study found an excess of depression in Cypriots but not in other groups, including African-Caribbean and Irish groups. This excess occurred despite the fact that they were less likely to live alone and were no more likely to be physically ill. They did, however, have increased subjective ill health and were much less likely to speak English or have had a secondary education and so were possibly less able to access help when required (Livingston et al, 2002). Cypriots with depression were likely to present to many services but not to complain of psychological symptoms. They often presented with prominent somatic symptoms. This is likely to be due to a different idiom of distress. Similar patterns of presentation were found in a study of immigrants to Israel from the former Soviet Union, with somatisation being more common in those who were older, currently single and female.

The vascular hypothesis of depression suggests that new depression in older age groups may be caused by cerebrovascular pathology (Hickie \& Scott, 1998) and therefore could be increased in the African-Caribbean population in the West. Older African-Caribbean people living in the West might therefore represent a population particularly at risk of depression from vascular causes and stroke. One recent study of older African-Caribbean people living in south London found an expected association of stroke and depression but no association with vascular risk factors in those who had not had a stroke (Stewart et al, 2001a). The conclusion was that it is stroke itself that causes depression rather than experiencing vascular risk factors.

The wide variation in rates of depression in those from the Indian subcontinent might reflect a complex picture. Any increased rates may be a result of comparing populations that differ in important confounding characteristics, such as physical ill health and social disadvantage. It might also be related to the use of inappropriate screening instruments. The results in studies on the prevalence of depressive disorders are therefore unsurprisingly inconsistent and inconclusive.

\section{Psychosis}

The issue of the overrepresentation in hospital of younger Black people with a diagnosis of schizophrenia has been studied extensively. This contrasts with the situation for older people, partly because there is less literature on psychosis and schizophrenia in this group. Depression and dementia in elders are more common and more studied than is schizophrenia. One study of paranoid symptoms in older people in the community reported that being Black was a risk factor (Blazer et al, 1996) but we did not find this in our study (Livingston et al, 2001b).

\section{Suicide}

Despite the high rate of completed suicide in older people, in particular older men, there has been little research considering the role of immigration and ethnicity as risk factors. Existing evidence is somewhat contradictory. In the UK, one study of suicide rates according to age and ethnicity found that all suicides in the over-75 age group were of Asian women and Whites of either gender and that there were no suicides from any other ethnic group (Neeleman et al, 1997). This contrasts with another report from the UK showing that suicide rates in elderly first-generation immigrants from the Indian subcontinent are low compared with those of the indigenous elderly population (Soni Raleigh et al, 1990). Although younger Asian women are more likely to self-harm than their White counterparts, this is clearly not so for the older Asian population (Bhugra et al, 1999). In the USA, the highest suicide rates were found to be among elderly White men (Cattell, 2000) . It has been suggested that older African Americans have surmounted more threats to their self-esteem than older Whites and are thereby better adapted to new challenges associated with ageing. However, older immigrants in Sweden have an increased rate of suicide (Johansson et al, 1997).

\section{Service utilisation}

The factors which influence service utilisation are complex and include accessibility, perception of usefulness, availability of alternative help, stigma and beliefs about racism. Thus, it is unclear how immigration will affect the use of services. 
Studies of immigrant Asian and Black elders showed that, in general, they were more likely to consult their general practitioner than their Britishborn counterparts. This contrasts with the picture of consultation in social service and secondary care. In general, the studies report findings on small numbers of older people and they are based on referrals. In the main, they suggest that ethnic elders are referred less (or have increased untreated morbidity) to secondary care health and social services, in particular psychiatric services, than their White UK-born counterparts (e.g. Shah \& Dighe-Deo, 1997). Recent studies, one comparing Asian and White referral to secondary care and one comparing service use by community-dwelling ethnic elders and by White counterparts, were unable to confirm this (e.g. Odutoye \& Shah, 1999; Livingston et al, 2002).

A variety of reasons has been suggested for any lack of utilisation, for example interpreting symptoms as a spiritual problem rather than as a depressive or physical illness. In addition, there have been reports of a reluctance in ethnic elders to accept referral to secondary mental health services (Shah et al, 1998) and of perceptions by Black people of racism in health workers (Hutchinson \& Gilvarry, 1998). It might be that the medical profession is less able to recognise the presentation of psychological distress by people from other cultures.

A recent qualitative study in London compared Black elders with and without depression with White counterparts. They found that Black elders often chose not to use medical services, as they perceived them to be irrelevant and stigmatising. There was, however, little evidence of perceived racism (Marwaha \& Livingston, 2003).

\section{Carers for those with dementia}

Caring for people with dementia is known to be stressful and associated with distress and depression, particularly in female and spouse caregivers. The meaning of caring and ways of coping may vary between ethnic and immigrant groups although the demands may be similar. Immigrants may have left their families and therefore their traditional sources of support. A review of 12 studies comparing White and non-White caregivers of people with dementia showed non-White caregivers to be less likely to be a spouse and more likely to be an adult child, friend or other family member. Non-White caregivers reported lower levels of caregiver stress, burden and depression. They endorsed more strongly held beliefs about filial support and were more likely to use prayer, faith or religion as a coping mechanism (Connell \& Gibson, 1997). This contrasts with a study comparing Hispanic and White non-Hispanic primary caregivers for dementia. This found that Hispanic caregivers were more likely to have depression, but the most robust risk factors were female gender and spouse status (Harwood et al, 1998).

\section{References}

Abas, M. A., Phillips, C., Carter, J., et al (1998) Culturally sensitive validation of screening questionnaires for depression in older African-Caribbean people living in south London. British Journal of Psychiatry, 173, 249-254.

Akpaffiong, M., Kunik, M., Hale, D., et al (1999) Crosscultural differences in demented geropsychiatric inpatients with behavioural disturbances. International Journal of Geriatric Psychiatry, 14, 845-850.

Bhatnagar, K. \& Frank, J. (1997) Psychiatric disorders in elderly from the Indian subcontinent living in Bradford. International Journal of Geriatric Psychiatry, 12, 907-912.

Bhugra, D., Desai, M. \& Baldwin, D. S. (1999) Attempted suicide in west London. I. Rates across ethnic communities. Psychological Medicine, 5, 1125-1130.

Blazer, D. G., Hays, J. C. \& Salive, M. C. (1996) Factors associated with paranoid symptoms in a community sample of older adults. Gerontologist, 36, 70-75.

Cattell, H. (2000) Suicide in the elderly. Advances in Psychiatric Treatment, 6, 102-108.

Connell, C. \& Gibson, G. (1997) Racial, ethnic and cultural differences in dementia caregiving: review and analysis. Gerontologist, 37, 355-364

Farrer, L. A., Cupples, L. A., Haines, J. L., et al (1997) Effects of age, sex and ethnicity on the association between apolipoprotein E genotype and Alzheimer disease. A meta-analysis. APOE and Alzheimer Disease Meta Analysis Consortium. JAMA, 278, 1349-1356.

Ganguli, M., Chandra, V., Kamboh, M. I., et al (2000) Apolipoprotein E polymorphism and Alzheimer disease: the Indo-US cross-national dementia study. Archives of Neurology, 57, 824-830.

Guglani, S., Coleman, P. \& Sonuga-Barke, E. J. S. (2000) Mental health of elderly Asians in Britain: a comparison of Hindus from nuclear and extended families of differing cultural identities. International Journal of Geriatric Psychiatry, 15, 1046-1053.

Hargrave, R., Stoeklin, M., Haan, M., et al (1998) Clinical aspects of Alzheimer's disease in Black and White patients. Journal of the National Medical Association, 90, 78-84.

Harwood, D., Barker, W., Cantillon, M., et al (1998) Depressive symptomatology in first-degree family caregivers of Alzheimer's disease patients: a cross-ethnic comparison. Alzheimer Disease and Associated Disorders, 12, 340-346.

Helman, C. G. (2000) Culture, Health and Illness. Oxford: Butterworth-Heinemann.

Hendrie, H. C., Ogunniyi, A., Hall, K. S., et al (2001) Incidence of dementia and Alzheimer disease in two communities: Yoruba residing in Ibadan, Nigeria, and African Americans residing in Indianapolis, Indiana. JAMA, 285, 739-747.

Hickie, I. \& Scott, E. (1998) Late-onset depressive disorders: a preventable variant of cerebrovascular disease? Psychological Medicine, 28, 1007-1013.

Hutchinson, G. \& Gilvarry, C. (1998) Ethnicity and dissatisfaction with mental health services (letter). British Journal of Psychiatry, 172, 95c-96c.

Johansson, L. M., Sundquist, J., Johansson, S. E., et al (1997) Suicide among foreign-born minorities and native Swedes: an epidemiological follow-up study of a defined population. Social Science and Medicine, 44, 181-187.

Jolley, D. \& Baxter, D. (1997) Mortality in elderly patients with organic brain disorder enrolled on the Salford psychiatric case register. International Journal of Geriatric Psychiatry, 12, 1174-1181.

Jorm, A. F., Korten, A. E. \& Henderson, A. S. (1987) The prevalence of dementia: a quantitative integration of the literature. Acta Psychiatrica Scandinavica, 76, 465-479. 
Lindesay, J. (1998) Diagnosis of mental illness in elderly people from ethnic minorities Advances in Psychiatric Treatment, 4, 219-226.

-, Jagger, C., Hibbett, M. J., et al (1997) Knowledge, uptake and availability of health and social services among Asian Gujarati and White elders. Ethnicity and Health, 2, 59-69.

Livingston, G., Leavey, G., Kitchen, G., et al (2001a) Mental health of migrant elders - the Islington study. British Journal of Psychiatry, 179, 361-366.

- Kitchen, G., Manela, M., et al (2001b) Persecutory symptoms and perceptual disturbance in a community sample of older people: the Islington study. International Journal of Geriatric Psychiatry, 16, 462-468.

-, Leavey, G., Kitchen, G., et al (2002) Accessibility of health and social services to immigrant elders: the Islington study. British Journal of Psychiatry, 180, 369-374.

Marwaha, S. \& Livingston, G. (2003) Racism, stigma or choice. Why do ethnic elders avoid psychiatrists. Journal of Affective Disorders, in press.

McCracken, C. F., Boneham, M. A., Copeland, J. R., et al (1997) Prevalence of dementia and depression among elderly people in Black and ethnic minorities. British Journal of Psychiatry, 171, 269-273.

Merril, J. \& Owens, J. (1988) Self-poisoning among four immigrant groups. Acta Psychiatrica Scandinavica, 77, 77-80.

Neeleman, J., Mak, V. \& Wessely, S. (1997) Suicide by age, ethnic group, coroner's verdict and country of birth. A three-year survey in inner London. British Journal of Psychiatry, 171, 463-467.

Odutoye, K. \& Shah, A. (1999) The characteristics of Indian subcontinent origin elders newly referred to a psychogeriatric service. International Journal of Geriatric Psychiatry, 14, 446-453.

Perkins, P., Annegers, J. F., Doody, R. S., et al (1997) Incidence and prevalence of dementia in a multiethnic cohort of municipal retirees. Neurology, 49, 44-50.

Rait, G., Burns, A. \& Chew, C. (1996) Age ethnicity and mental illness: a triple whammy. BMJ, 313, 1347.

- - - Baldwin, R., et al (2000) Validating screening instruments for cognitive impairment in older South Asians in the United Kingdom. International Journal of Geriatric Psychiatry, 15, 54-62.

Richards, M., Brayne, C., Dening, T., et al (2000) Cognitive function in UK community-dwelling African Caribbean and white elders: a pilot study. International Journal of Geriatric Psychiatry, 15, 621-630.

Schofield, P. W., Tang, M., Marder, K., et al (1995) Consistency of clinical diagnosis in a community based longitudinal study of dementia and Alzheimer's Disease. Neurology, 45, 2159-2164.

Shah, A., Lindesay, J. \& Jagger, C. (1998) Is the diagnosis of dementia stable over time in elderly immigrant Gujaratis in the UK (Leicester). International Journal of Geriatric Psychiatry, 7, 440-444.

Shah, A. K. \& Dighe-Deo, D. (1997) Elderly Gujaratis and psychogeriatrics in a London psychogeriatric service. Bulletin of the International Psychogeriatric Association, 14, 12-13.

Silveira, E. \& Ebrahim, S. (1998a) A comparison of mental health among minority ethnic elders and Whites in East and North London. Age and Ageing, 27, 375-383.

— \& - (1998b) Social determinants of psychiatric morbidity and well-being in immigrant elders and Whites in east London. International Journal of Geriatric Psychiatry, 13, 801812

Soni Raleigh, V., Bulusu, L. \& Balarajan, R. (1990) Suicides among immigrants from the Indian subcontinent. British Journal of Psychiatry, 156, 46-50.

Stewart, R., Prince, M., Mann, A., et al (2001a) Stroke, vascular risk factors and depression: cross-sectional study in a UK Caribbean-born population. British Journal of Psychiatry, 178, 23-28.

-, Richards, M., Brayne, C., et al (2001b) Cognitive function in UK community-dwelling African Caribbean elders: normative data for a test battery. International Journal of Geriatric Psychiatry, 16, 518-527.

\section{Multiple choice questions}

1 With regard to the mental health of ethnic elders:

a immigrant elders are concentrated in rural areas

b $15 \%$ of older people in the UK are immigrants

c ethnicity is self-defined

$\mathrm{d}$ ethnicity is fixed

e the number of immigrant elders is increasing.

2 With regard to the diagnosis of dementia in immigrant ethnic elders:

a diagnosis is both difficult and less stable than in White counterparts

b there is evidence of a higher prevalence of dementia in Black African-Caribbean immigrants

c there is evidence of a higher prevalence of dementia in older people living in Nigeria

$d$ the APOE 4 allele frequency varies between Black and White Americans

e ethnic status affects the mortality rate in dementia.

3 With regard to depression in immigrant elders:

a depression presents with different patterns of symptoms in different cultures

b when screening for depression, higher cut-points are appropriate for older Black people in the UK

c depression in older Black people is associated with vascular risk factors apart from stroke

d Irish older people have higher rates of depression

e reported prevalence rates of depression in older people from the Indian subcontinent vary widely.

4 Concerning elderly immigrants:

a non-White carers are more likely to be a spouse than White carers

b there are higher levels of carer stress and depression in the Hispanic population

c carers rarely use religion/prayer as a source of support

d older immigrants in Sweden have an increased risk of suicide

e elderly Asian women living in the UK show a consistently higher rate of suicide than their indigenous counterparts.

5 The following reasons may account for immigrant elders' underutilisation of psychiatric services:

a symptom interpreted as a spiritual problem

$b$ lower rates of illness

c decreased utilisation of primary care services

d perceived racism in services

e symptoms perceived as a physical problem.

\section{MCQ answers}

\begin{tabular}{|c|c|c|c|}
\hline 1 & 2 & 3 & 4 \\
\hline $\mathrm{F}$ & a $F$ & a $\mathrm{T}$ & a $F$ \\
\hline $\mathrm{F}$ & $\mathrm{b} \quad \mathrm{T}$ & $b \quad F$ & b $\mathrm{T}$ \\
\hline $\mathrm{T}$ & c F & c F & C F \\
\hline $\mathrm{F}$ & d F & d F & d $\mathrm{T}$ \\
\hline $\mathrm{T}$ & e F & e $\mathrm{T}$ & e F \\
\hline
\end{tabular}

